\title{
Time-variability and disk geometry in Herbig Ae/Be disks
}

\author{
R. Szakáts ${ }^{1}$, P. Ábrahám ${ }^{1}$, Á. Kóspál ${ }^{1,2}$ \\ ${ }^{1}$ Konkoly Observatory, Research Centre for Astronomy and Earth Sciences, Hungarian \\ Academy of Sciences, Konkoly-Thege Miklós út 15-17, H-1121, Budapest, Hungary \\ ${ }^{2}$ Max Planck Institute for Astronomy, Königstuhl 17, D-69117 Heidelberg, Germany \\ email: szakats.robert@csfk.mta.hu
}

\begin{abstract}
The origin of optical-infrared variability in young, intermediate mass Herbig Ae/Be stars is linked to their circumstellar disk. Therefore, variability could serve as a diagnostic tool to constrain the structure and dynamics of the (inner) disk. Here we discuss this diagnostic potential, and report some preliminary results from our coordinated $B V R I J H K_{S}$ and Spitzer monitoring observations of nine Herbig Ae stars. We aim to understand the response of the inner disks thermal emission on the changing stellar irradiation, and to separate it from UX Orionistype fading events, which also provide information on the disk. This project is a pilot study for the era of time domain astronomy of young stars, opened by Kepler K2, Gaia, ASAS-SN, TESS, Spitzer, WISE, and JWST.
\end{abstract}

Keywords. stars: pre-main-sequence, circumstellar matter

\section{Motivation: linking variability with disk structure}

The three main physical processes causing variability are all connected to the disk: (a) fluctuating mass accretion from the disk changes the luminosity of the central source (optical light curve), and leads to variable irradiation of the disk (infrared light curve); b) variable emitting surface of the disk, e.g. by temporary dust clouds above the disk surface, increase the thermal infrared emission; and c) UX Ori (UXor)-type eclipses, when dust clumps in the inner disk pass through the line-of-sight. Radiative transfer models quantitatively predict the wavelength dependent light curves (Kesseli et al. 2016). Observationally, we looked for a correlation between the variability amplitude at $6-8 \mu \mathrm{m}$ (Kóspál et al. 2012) and the disk flaring (Meeus et al. 2001). We found higher amplitudes in flatter (Group IIa) disks. Plotting the same variability amplitudes wrt. the radius of the interferometric emitting area (Menu et al. 2015) implies that variability in disks with more centrally concentrated brightness distribution is stronger. These results imply a physical link between variability and disk structure, and suggest that variability may provide information about the disk structure and dynamics on the smallest spatial scales, a few au from the star.

\section{The Konkolyvar project}

In order to decide which physical processes are responsible for the observed variability in a particular system, and to deduce disk properties, simultaneous optical-infrared observations are needed. We carried out a 2-week-long quasi-simultaneous optical-infrared photometric monitoring program with daily cadence, in 2009 Oct-Nov. We targeted nine Herbig Ae stars and collected observations from Piszkéstető Observatory (Hungary), Teide Observatory (Spain), and at 3.6 and $4.5 \mu \mathrm{m}$ from the Spitzer Space Telescope. 

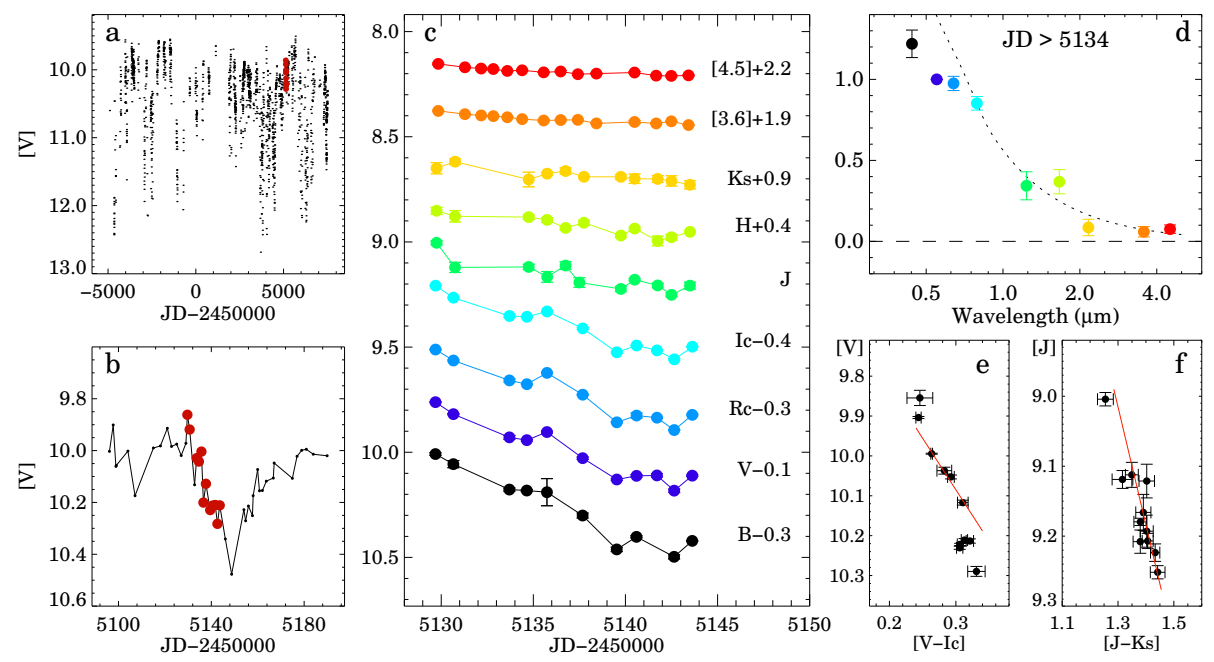

Figure 1. Multiwavelength light curves for BF Ori from the Konkolyvar project.

\section{The physical origin of the variability, and the response of the disk}

As an example, Fig. 1 presents our observations for BF Ori, where panel $c$ shows the multiwavelength light curves. Comparison with earlier/later $V$-band observations (panel $b$ ) outlines that our first two epochs coincided with a small brightness peak, while later the source entered a deep minimum. Panels $d, e$, and $f$ imply that the fading occurred along the extinction path, and was probably a typical UXor event. The faintest data points exhibit optical colors deviating from the extinction curve: here we see the well-known blueing of UXors, caused by scatter light.

We also compared the Spitzer 3.6/4.5 $\mu \mathrm{m}$ data with the $I$-band light curve. If the whole inner disk was obscured in an UXor event, shapes of the $I$-band and Spitzer light curves would be identical, apart from a lower amplitude in the mid-infrared according to the extinction law (e.g., Cardelli et al. 1989). This is the case for BF Ori at JD > 2455135, but the first few points exhibit the response of the disk with unexpectedly high amplitude for the small V-band brightness peak. Analysing the other stars from the Konkolyvar sample we found large variety: in RR Tau most variability is related to extinction changes, while in UX Ori the disk emission is virtually unrelated to the optical flux changes, because in the second part of our campaign the optical flux increases while the mid-infrared flux drops. The detailed analysis of these results in terms of disk geometry and dynamics is ongoing.

Acknowledgements. This project has received funding from the European Research Council (ERC) under the European Union's Horizon 2020 research and innovation programme under grant agreement No 716155 (SACCRED). This work is based in part on observations made with the Spitzer Space Telescope, which is operated by the Jet Propulsion Laboratory, California Institute of Technology under a contract with NASA.

\section{References}

Cardelli, J.A., Clayton, G.C., Mathis, J.S. 1989, ApJ, 345, 245

Kesseli, A.Y., Petkova, M.A., Wood, Kenneth et al. 2016, ApJ, 828, 42

Kóspál, Á., Ábrahám, P., Acosta-Pulido, J.A. et al. 2012, ApJ Suppl. Ser., 201, 11

Meeus, G., Waters, L.B.F.M., Bouwman, J. et al. 2001, $A \& A, 365,476$

Menu, J., van Boekel, R., Henning, Th. et al. 2015, A\&\&A, 581, A107 